\title{
Identification Of Three Key Factors Contributing To The Aetiology Of Dark Circles By Clinical And Instrumental Assessments Of The Infraorbital Region
}

This article was published in the following Dove Press journal: Clinical, Cosmetic and Investigational Dermatology

\author{
Sophie Mac-Mary ${ }^{\prime}$ \\ Itziar Zornoza Solinis ${ }^{2}$ \\ Océane Predine' \\ Jean-Marie Sainthillier' \\ Christelle Sladen ${ }^{2}$ \\ Mike Bell $\mathbb{D D}^{2}$ \\ Mark O'Mahony ${ }^{2}$ \\ 'Skinexigence, Besançon, France; \\ ${ }^{2}$ Walgreens Boots Alliance, \\ Nottingham, UK
}

Correspondence: Sophie Mac-Mary Skinexigence SAS, 2 Rue Paul Milleret, 25000, Besançon, France

$\mathrm{Tel}+33381889551$

Email smac@skinexigence.com
Background: The aetiology of infraorbital dark circles is complex and multi-factorial. The aim of this research was to measure and characterize dark circles and to determine the physiological changes associated with the occurrence of this aesthetically unpleasing issue. Materials and methods: Clinical, photographic and instrumental assessments were performed on Caucasian skin to determine the most appropriate methodologies to measure dark circles, comparing different zones of the infraorbital region in subjects with and without dark circles. Exploratory studies were also carried out on African and Far East Asian skin, as well as on tracking the natural variation of dark circles over seven days in Caucasian subjects.

Results: Under-eye dark circles in Caucasian subjects are characterized by significantly darker coloured skin and higher colour deviations between the dark circle region and surrounding areas of skin. Multispectral image analysis produced a higher melanin index in subjects with dark circles, suggesting hyperpigmentation in the affected area, in addition to a higher haemoglobin index. The higher haemoglobin index, combined with preliminary assessments of the vascular network by videocapillaroscopy, suggests there may be more dilated, thicker or increased numbers of capillaries in subjects with dark circles. Ultrasound analysis showed a significant difference in skin thickness between the two groups of subjects linking the appearance of dark circles to thinner skin under the eye. Many of these trends were also observed in African and Far East Asian skin. The results also showed intra-individual, intra-day and inter-day variability of dark circle intensity in subjects with Caucasian skin.

Conclusion: Three key physiological factors associated with the occurrence of infraorbital dark circles are hyperpigmentation, a tendency for more dilated, thicker or increased number of capillaries and thinner skin in the under-eye area. The combination of these three factors provides a robust indication of the presence of infraorbital dark circles.

Keywords: dark circles, infraorbital, ultrasound imaging, image processing, multispectral imaging, pigmentation

\section{Introduction}

Infraorbital dark circles are generally not a cause of medical concern; however, they are aesthetically unpleasing for many individuals, particularly for women, and can occur across all age brackets. ${ }^{1}$ Their aetiology is complex and multi-factorial, and there are numerous intrinsic and extrinsic factors associated with their occurrence. Visually, dark circles are characterized by differences in colour between the palpebral and surrounding areas of facial skin. The colour of the palpebral skin is strongly associated with intrinsic factors, such as an individual's ethnic origin and genetics, including the amount and 
quality of melanin pigment in the skin. However, extrinsic and lifestyle factors also play a crucial role. ${ }^{2-7}$

Dark circles are most often associated with tiredness, sleep deprivation and fatigue; however, there are numerous other extrinsic and lifestyle-related triggers, including illness/infections, atopy and allergies, stress, hormonal changes, chronic irritation of the eye area, eye strain, exposure to UV light, dehydration, poor nutrition, vitamin deficiency, excessive alcohol consumption and smoking. Certain medicines, such as non-steroidal anti-inflammatory drugs and chemotherapy drugs, are also suspected to trigger the occurrence of dark circles. $^{7-11}$ The anatomy of the eye area is complex and can also be a contributory factor, for example, there is minimal infraorbital subcutaneous fat and the area is prone to blood stasis and oedema. In addition, the eye-lids have the thinnest skin of the face, and the bone structure and contour of the eye socket can lead to shadowing effects around the eye. ${ }^{1,12,13}$

In view of all these various factors, dark circles are difficult to characterize and measure. While many articles detail the origins and triggers of dark circles, there are only a few that describe how to assess and characterize them. ${ }^{14,15}$ The aim of this work was to assess different techniques to discriminate between subjects with and without dark circles, to measure and quantify dark circles across different ethnic skin tones, to assess their episodic nature and to determine the physiological changes associated with their occurrence.

\section{Materials And Methods}

\section{Subject Recruitment}

Subjects were recruited with dark circles or without dark circles based on assessment of the eye region by an experienced technician. Inclusion and exclusion criteria are listed in Table 1. Subjects with dark circles were required to have dark or very dark areas towards the inner corner of the eye. Before enrolment in the studies, written informed consent was collected from all subjects and the studies were conducted in accordance with the Declaration of Helsinki principles (2013). All assessments were performed in a controlled environment $\left(22 \pm 2^{\circ} \mathrm{C}\right.$ and $50 \pm 10 \%$ Relative Humidity) on skin where no product or make-up had been applied since the evening before the visit. Subjects remained at rest in the examination room for 30 mins before assessments to ensure blood flow and skin temperature were stable. The assessments showed that many subjects appeared to have periorbital dark circles, with both under and over the eye affected, but given that the over eye area is less visible when the eyes are open, and therefore less of an aesthetic concern, we focused our assessment on the infraorbital area. Assessments were performed on three distinct zones along the infraorbital dark circle, as well as on a control zone on the cheek (Figure 1A). Additionally, for some colour assessments, a control zone on the forehead was also selected.

\section{Clinical And Instrumental Measurements To Refine Infraorbital Measurement Areas And Techniques}

Twenty-four healthy women with Caucasian skin (Phototypes I to III) were included in a preliminary study $(\mathrm{N}=12$ without dark circles, age $32.7 \pm 9.2 ; \mathrm{N}=12$ with dark circles, age $30.2 \pm$ 8.7) with the aim of determining appropriate techniques and the most reproducible infraorbital locations for measuring dark circles. Assessments consisted of in-situ clinical scoring of the intensity of the dark circle in each of the three zones under the eye by an experienced technician under standardized lighting using a six-point scoring scale $(0=$ absence, $1=$ =very light, $2=$ light, $3=$ moderate, $4=$ dark, $5=$ very dark) and colour analysis

Table I Inclusion And Exclusion Criteria For Subject Recruitment

\begin{tabular}{|c|c|}
\hline Inclusion & Exclusion \\
\hline $\begin{array}{l}\text { Female } \\
\text { I8-55 years old } \\
\text { Willing to sign a written informed consent } \\
\text { Phototypes I to III for Caucasian } \\
\text { or African skin } \\
\text { or Far East Asian skin } \\
\text { With persistent orbital dark circles, defined as having an intensity } \\
\text { rated as dark or very dark at the inner corner of the eye and an } \\
\text { extension of a minimum of } 35 \% \text { across the infraorbital area } \\
\text { or without dark circles }\end{array}$ & $\begin{array}{l}\text { Pregnant, nursing or intending to become pregnant during the study } \\
\text { Marked eyes bags and/or marked wrinkles under the eyes } \\
\text { Ocular pathology and/or skin disease likely to interfere with the measured } \\
\text { parameters } \\
\text { Topical or systemic treatment which could interfere with the results of the } \\
\text { study } \\
\text { Sleep disorders } \\
\text { History of epilepsy } \\
\text { Aesthetical or dermatological treatment that may interfere with the study } \\
\text { Exposure to UV in the } 3 \text { weeks prior to the study and/or intended facial UV } \\
\text { exposure during the study }\end{array}$ \\
\hline
\end{tabular}


A

Without Dark Circles

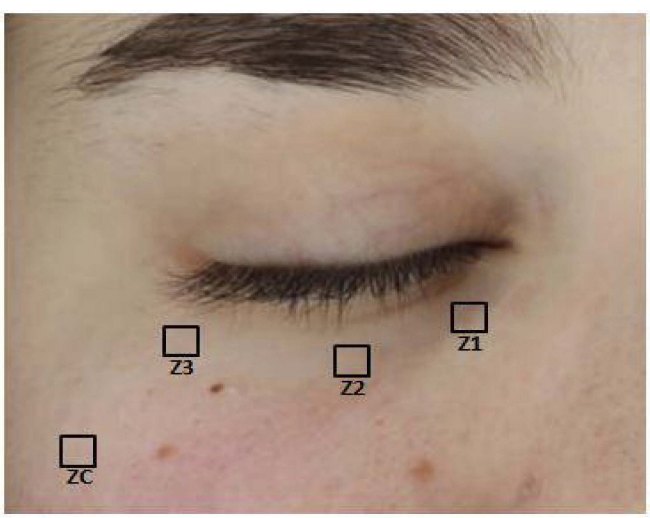

With Dark Circles

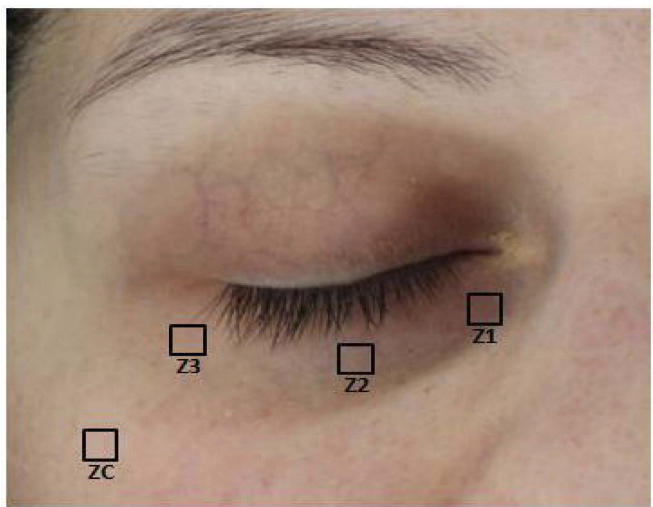

B

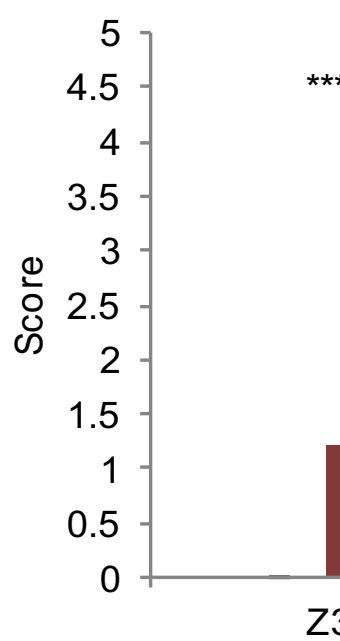

\section{Clinical Scoring}

$\star \star \star$

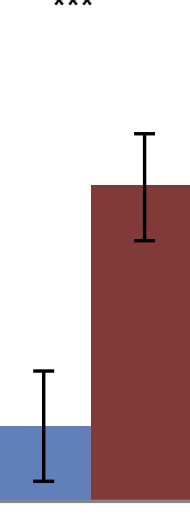

Z2
$* * *$

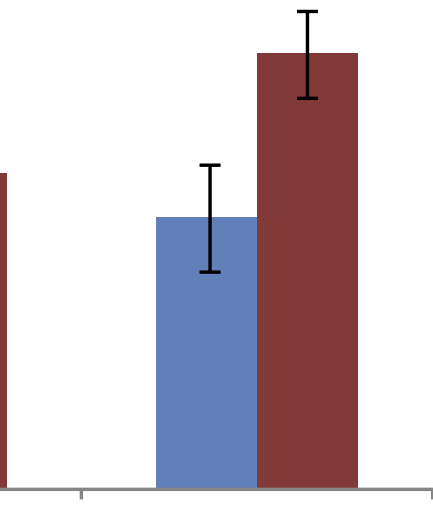

Z1

Without Dark Circles $\quad$ With Dark Circles

C
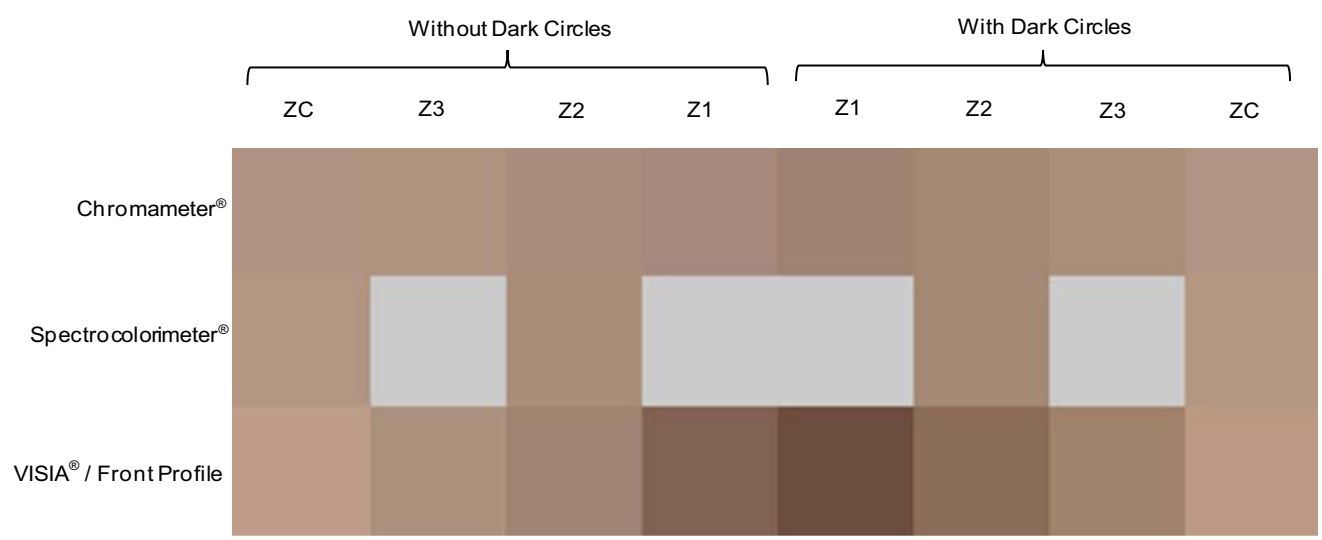

Figure I (A) Images captured with the VISIA-CR of subjects with and without dark circles, showing the position of the infraorbital zones and the control zone on the cheek. (B) Clinical scoring of dark circle intensity in the three zones under the eye. ( $\mathrm{N}=12$ with dark circles versus $\mathrm{N}=12$ without dark circles, $* * * p<0.00 \mathrm{I}$ ). (C) Comparison of the mean colorimetric data ( $\mathrm{L}^{*} \mathrm{a}^{*} \mathrm{~b}^{*} \mathrm{CIELAB}$ 1976) assessed with Chromameter, Spectrocolorimeter and VISIA image processing. (Grey = measurement not taken). Abbreviations: ZI, Zone I; Z2, Zone 2; Z3, Zone 3; ZC, Cheek Control Zone. 
(L*a*b* CIELAB 1976; L*Lightness; a*red-green component; $b^{*}$ yellow-blue component) of the three zones, plus the control zone on the cheek, using chromametry (CR-400, Konica Minolta, Japan) and image processing of cross-polarized facial photographs captured with the VISIA-CR (Canfield, USA). Spectrocolorimetry analysis (CM2600-d, Konica Minolta, Japan) was performed on Z2 and the cheek only, as the equipment was difficult to handle in the under-eye area.

\section{Clinical And Instrumental Measurements Of Dark Circles On Caucasian Skin}

Using insights from the preliminary study, a second study was performed on a further 24 women with Caucasian skin (Phototypes II and III); $\mathrm{N}=12$ without dark circles, age $33.2 \pm 9.2 ; \mathrm{N}=12$ with dark circles, age $34.9 \pm 8.6$. Clinical scoring of the intensity of the dark circles and colour analysis using image processing of facial photographs were repeated. A number of additional assessments were also performed in this study including ultrasound imaging (Dermcup, Atys, France) to determine skin thickness, analysis of the under-eye vascular network by videocapillaroscopy (MS-500, Moritex, Japan) and multispectral imaging (VideometerLab Agile, Videometer, Denmark). Ultrasound imaging was performed at a frequency of $20 \mathrm{MHz}$. At least three images were acquired from each area of interest, and analysis was performed on three parts of the image to obtain epidermal/dermal thickness. For multispectral analysis, L*a*b*, haemoglobin (HI) and melanin (MI) indices were assessed and complemented by a normalized canonical discriminant analysis (nCDA) of the overall dark circles area. nCDA was carried out from the multispectral cubes. This example-based analysis consists of manually marking multiple points on an area of prominent dark circle and on areas of skin without dark circles. The resulting pixels from both classes are then used to develop a discriminant function that maximizes their separation. The result of this classification is an image where all the points are between 0 (the probability of dark circle is null) and 1 (the probability of dark circle is certain), and the dark circles detector was refined by combining the marked points of three different faces. Using the same principle, indices of melanin and haemoglobin were also developed.

\section{Clinical And Instrumental Measurements Of Dark Circles On African And Far East Asian Skin And Assessment Of The Natural Variation Of Dark Circles}

Exploratory studies were carried out to assess ethnic differences in the aetiology of dark circles and to assess the episodic natural variation of dark circles. A small number of subjects with African skin (Phototype VI, $\mathrm{N}=6$ without dark circles, age $36.2 \pm 10.6 ; \mathrm{N}=9$ with dark circles, age $28.9 \pm 12.4$ ) and with Far East Asian skin ( $\mathrm{N}=7$ without dark circles, age $31.4 \pm$ $3.7 ; \mathrm{N}=4$ with dark circles, age $38.8 \pm 5.4$ ) were recruited and a selection of the assessments performed on Caucasian skin were repeated in these groups. For episodic evaluation of the dark circles, nine women with Caucasian skin (age $32.8 \pm 9.4$ ) were studied over seven days. Clinical scoring of intensity, facial photography combined with image processing and multispectral image analysis were performed in the morning and afternoon of day 0 , day 4 and day 7 . To assess the variation, minimum and maximum values for each parameter were compared for each subject over all visits. To assess intra-day and inter-day variations, comparisons were performed on the mean of the maximum delta between morning and afternoon on each of the three days and the mean of the delta between the maximum and minimum day averages.

\section{Data Analysis}

Descriptive statistics were determined for each parameter including number of observations, mean, standard deviation, minimum, maximum, median and quartiles. Normality of the distribution of the data sets was calculated using the Shapiro-Wilk test, and subsequently analysis was performed using either the Student test or the Wilcoxon test to determine statistical differences between subjects with and without dark circles. Matlab R2014a with statistical toolbox was used to carry out multivariate analysis.

\section{Results}

\section{Refinement Of Infraorbital Measurement Areas And Techniques}

Clinical scoring of intensity in the three zones under the eye (Figure 1A) revealed significant differences between Caucasian subjects with and without dark circles (Figure 1B), with the score decreasing as the zone moved laterally away from the inner corner of the eye. The results also clearly indicate that there is darkening in the inner zone of the eye (Z1) even in subjects without evident dark circles. 
Colour analysis using chromametry and image processing of cross-polarized facial photographs captured with the VISIACR was consistent with the clinical scoring, showing a colour gradient along the three zones of the dark circle (Figure 1C) and significant differences $(\mathrm{p}<0.05)$ in $\mathrm{L}^{*}$ in $\mathrm{Z1}$ and $\mathrm{Z} 2$ between those with and without dark circles. Only Z2 was measured using the spectrocolorimeter, as it was difficult to handle in the under-eye area, and while the colour difference was less distinct (Figure 1C), a significant difference in $\mathrm{L}^{*}$ between the two groups of subjects was revealed $(p<0.01)$. Correlation analysis was subsequently performed between clinical scores and the instruments used for colour analysis. Significant correlations were observed in Z2 whatever the technique, though not for all colour parameters (Table 2). Furthermore, correlations were higher between clinical scoring and $\mathrm{L}^{*}$ and, in general, were stronger for results generated using image processing of VISIA photographs, than for chromametry or spectrocolorimetry. Multivariate analysis also indicated that $\mathrm{Z} 2$ was the best zone to assess dark circles, particularly for colour parameters. The results of this study directed subsequent phases of research, where image processing of VISIA facial photographs was the primary technique used for colour discrimination of subjects with and without dark circles, and certain additional techniques were performed only on Z2.

\section{Assessment Of Dark Circles On Caucasian Skin}

In the second phase of research, clinical scoring results confirmed the significant differences observed in phase one on Caucasian skin across all three infraorbital zones in subjects with and without dark circles. Colour analysis of VISIA images showed that infraorbital dark circles are characterized by significantly darker coloured skin $\left(\mathrm{L}^{*}\right)$ across all three zones and a higher colour difference (DeltaE) between the dark circles region and the surrounding area of cheek skin (Figure 2A and B). No significant colour difference was detected between skin on the cheek and forehead (data not shown). In addition, this data suggest that subjects with dark circles tend to have significantly more red colour in this region (increased $\mathrm{a}^{*}$ ), as well as a trend towards more yellow colour (increased $b^{*}$ ), although this was less clear, and it appears that subjects with dark circles also have more yellow colour in the control zone on the cheek (Figure 2C and D). Significant correlation was again observed between clinical scores and $\mathrm{L}^{*}$ values across all three zones (data not shown).

In this second phase, additional techniques were employed to further characterize the under-eye area. Multispectral images were captured using the VideometerLab Agile, and colour analysis was performed. The results obtained for $\mathrm{L}^{*} \mathrm{a} \mathrm{b}^{*}$ analysis were similar to those obtained with image processing of VISIA images (data not shown), suggesting that colour analysis, particularly $\mathrm{L}^{*}$ is a valid technique to discriminate between subjects with and without dark circles. Multispectral imaging also allowed determination of melanin (MI) and haemoglobin (HI) indices, as well as discrimination of the overall dark circle. The images clearly show a visual difference between subjects with and without dark circles (Figure 3). Analysis of the overall dark circle showed significant differences between the two groups (Figure 3A). Furthermore, image analysis suggests that subjects with dark circles have more melanin and haemoglobin in the under-eye area, although subjects with dark circles also tended to have

Table 2 Instrumental Measurements Of Colour Parameters In Zone 2 ( \pm Standard deviation) And Pearson Correlation Between Clinical Scoring And Colour Parameters Of Z2 In Caucasian Subjects, Calculated By Combining The Data From Subjects With And Without Dark Circles

\begin{tabular}{|c|c|c|c|c|}
\hline Instrument & $\begin{array}{l}\text { Colour Parameter } \\
\text { Z2 }\end{array}$ & $\begin{array}{l}\text { Without Dark } \\
\text { Circles }\end{array}$ & $\begin{array}{l}\text { With Dark } \\
\text { Circles }\end{array}$ & $\begin{array}{l}\text { Pearson Correlation Coefficient } \\
\text { Clinical Scoring vs Colour Measurements } \\
\text { of Z2 }\end{array}$ \\
\hline Chromameter & $\begin{array}{l}\mathbf{L}^{*} \\
\mathbf{a}^{*} \\
\mathbf{b}^{*}\end{array}$ & $\begin{array}{l}61.8 \pm 1.4 \\
14.2 \pm 1.99 \\
14.2 \pm 1.6\end{array}$ & $\begin{array}{l}59.6 \pm 1.2 \\
12.4 \pm 1.4 \\
15.4 \pm 1.3\end{array}$ & $\begin{array}{l}r=-0.59(p<0.05) \\
\text { ns } \\
\text { ns }\end{array}$ \\
\hline Spectrocolorimeter & $\begin{array}{l}L^{*} \\
a^{*} \\
b^{*}\end{array}$ & $\begin{array}{l}62.4 \pm 1.1 \\
12.3 \pm 1.1 \\
15.4 \pm 1.4\end{array}$ & $\begin{array}{l}60.4 \pm 1.5 \\
11.6 \pm 1.0 \\
16.5 \pm 1.3\end{array}$ & $\begin{array}{l}r=-0.53(p<0.05) \\
\text { ns } \\
r=0.53(p<0.05)\end{array}$ \\
\hline VISIA Front Profile & $\begin{array}{l}L^{*} \\
a^{*} \\
b^{*}\end{array}$ & $\begin{array}{l}58.6 \pm 5.2 \\
11.5 \pm 2.9 \\
15.3 \pm 3.4\end{array}$ & $\begin{array}{l}49.9 \pm 6.5 \\
14.6 \pm 2.8 \\
18.3 \pm 3.0\end{array}$ & $\begin{array}{l}r=-0.62(p<0.05) \\
r=0.57(p<0.05) \\
r=0.50(p<0.05)\end{array}$ \\
\hline
\end{tabular}

Abbreviations: r, Pearson correlation coefficient; ns, not significant; L*, lightness; a*, red-green component; b*, yellow-blue component; Z2, Zone 2 . 
A



\section{Without Dark Circles $\quad$ With Dark Circles}

C



B

VISIA Image Processing DeltaE

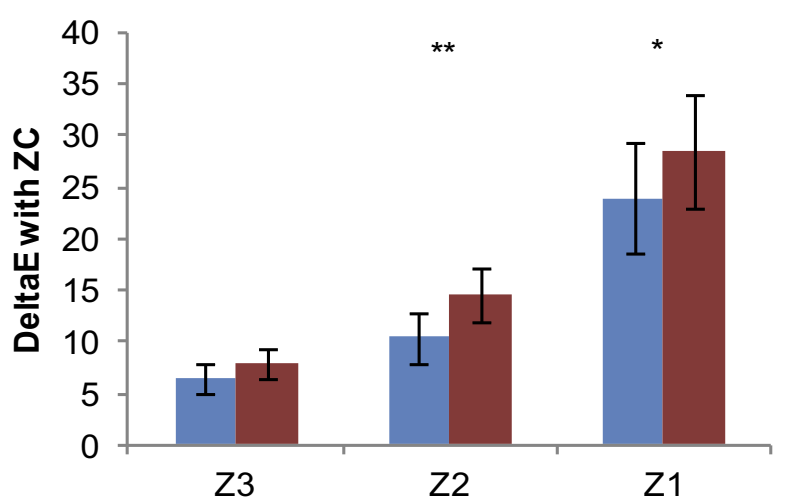

Without Dark Circles $\quad$ With Dark Circles

D

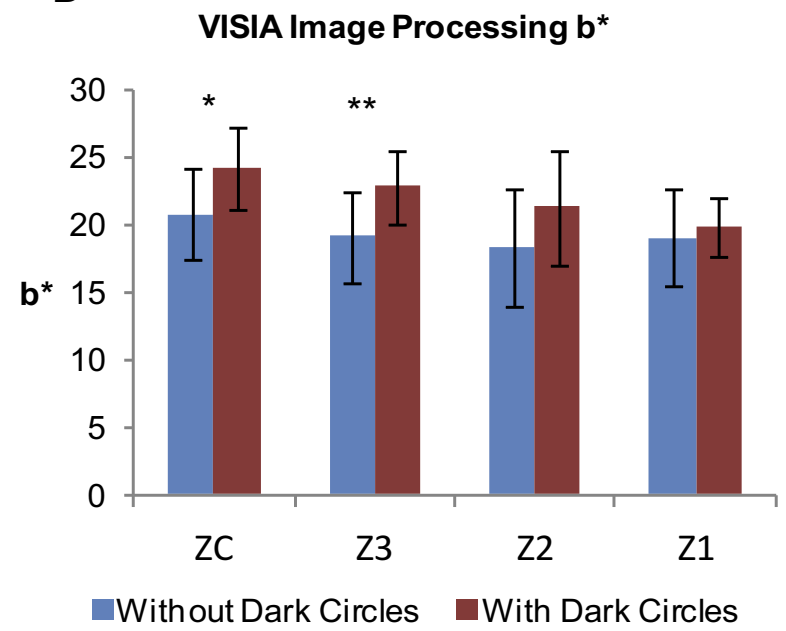

Figure 2 Colour analysis of the zones by image processing of VISIA photographs; (A) L*Lightness. (B) DeltaE, Colour difference between each of the infraorbital zones and the cheek. (C) a*red-green component. (D) b*yellow-blue component. $\left(\mathrm{N}=12\right.$ with dark circles versus $\mathrm{N}=12$ without dark circles, ${ }^{*} \mathrm{p}<0.05$, $\left.* * \mathrm{p}<0.0 \mathrm{I}\right)$.

Abbreviations: ZI, Zone I; Z2, Zone 2; Z3, Zone 3; ZC, Cheek Control Zone.

more melanin in the cheek area, suggesting overall higher levels of melanin (Figure 3B).

Ultrasound imaging of Z2 showed a significant difference in epidermal/dermal skin thickness between subjects with and without dark circles, suggesting that dark circle appearance is linked to thinner skin under the eye (Figure 4). Again, a significant correlation was observed between measurements of skin thickness by ultrasound and clinical scoring $(\mathrm{r}=-0.43)$. No significant difference was detected on cheek skin between those with dark circles and those without.

Assessment of the under-eye vascular network by videocapillaroscopy suggested that subjects with dark circles tended to have more dilated, thicker or increased numbers of capillaries (Figure 5). Visual differences between the two groups of subjects were clear, but further work is required to quantitatively confirm the differences observed.

The above instrumental measurements assessing Caucasian subjects with and without dark circles are summarised in Table 3.

\section{Assessment Of Dark Circles On African And Far East Asian Skin}

Results of the exploratory studies on African and Far East Asian skin were similar to those observed for Caucasians, though because of the lower number of subjects the differences observed were not always significant. Clinical scoring of intensity did show significant differences in $\mathrm{Z} 1$ and $\mathrm{Z} 2$ for both African and Far East Asian skin with and without dark 
A

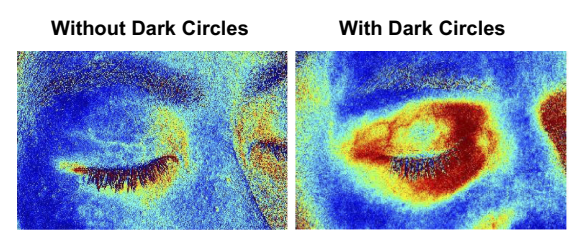

Multispectral Imaging Dark Circle nCDA

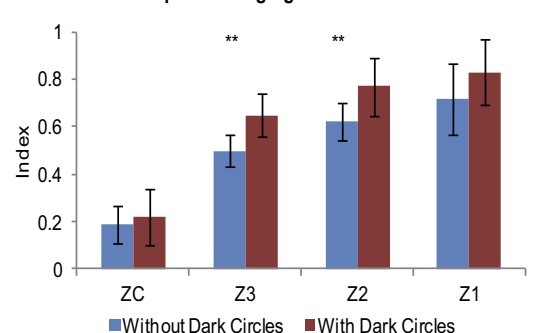

B

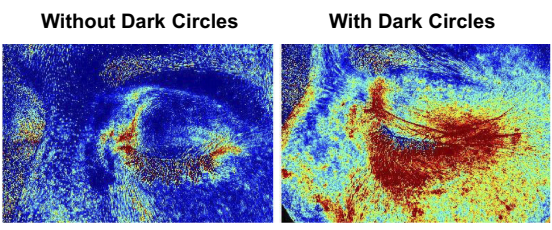

Multispectral Imaging Melanin Index

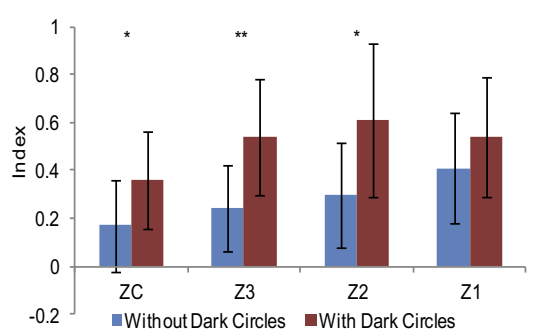

C

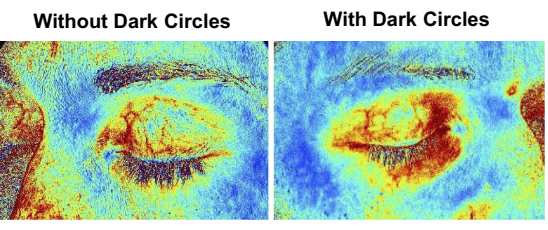

Multispectral Imaging Hemoglobin Index

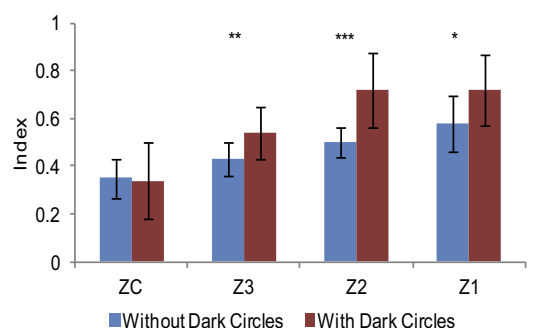

Figure 3 Multispectral analysis of the infraorbital zones including example images of $(\mathbf{A})$ overall dark circle, $(\mathbf{B})$ melanin, and $(\mathbf{C})$ haemoglobin. $(\mathrm{N}=12$ with dark circles versus $\mathrm{N}=12$ without dark circles, ${ }^{*} \mathrm{p}<0.05,{ }^{* *} \mathrm{p}<0.01$, $\left.{ }^{* * *} \mathrm{p}<0.00 \mathrm{I}\right)$.

Abbreviations: ZI, Zone I; Z2, Zone 2; Z3, Zone 3; ZC, Cheek Control Zone; nCDA, normalized canonical discriminant analysis.

A
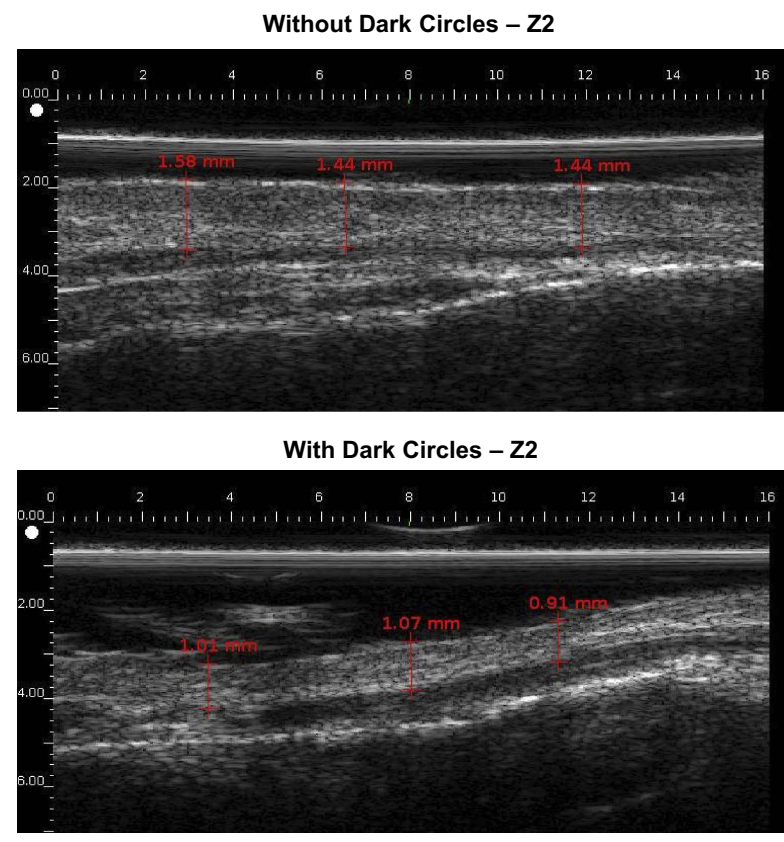

B

Skin Thickness

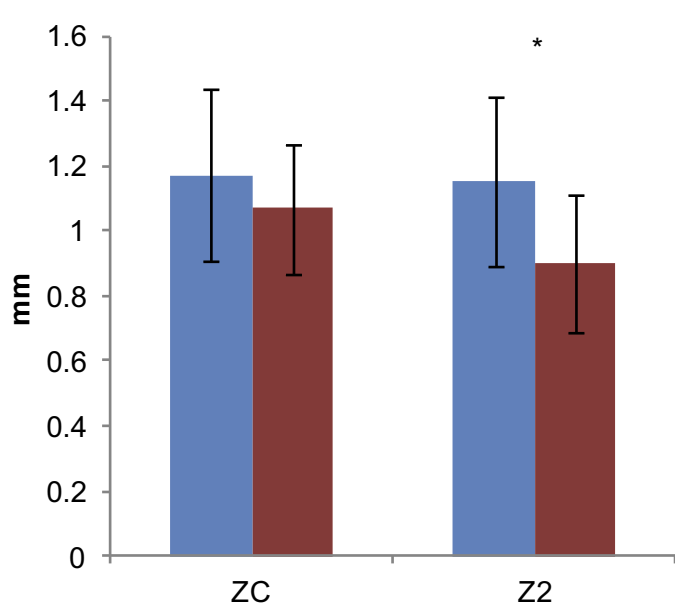

Without Dark Circles $\quad$ With Dark Circles

Figure 4 Ultrasound analysis; (A) Example ultrasound images from subjects with and without dark circles. The red lines indicate epidermal/dermal thickness measurements. (B) Average skin thickness measurements across both populations. $\left(\mathrm{N}=12\right.$ with dark circles versus $\mathrm{N}=12$ without dark circles, $\left.{ }^{*} \mathrm{p}<0.05\right)$.

Abbreviations: ZC, Cheek Control Zone; Z2, Zone 2.

circles. Colour analysis showed similar trends to those observed in Caucasian skin, particularly for the DeltaE parameter and multispectral imaging also suggests there may be more melanin and haemoglobin in subjects with dark circles. For skin thickness, differences were observed for African and Far East Asian skin, and when all three populations were combined, there was an overall significant difference between subjects with and without dark circles. Further work is required to fully characterize dark circles in African and Far East Asian skin.

\section{Assessment Of The Natural Variation Of Dark Circles}

The final phase of research focused on assessing the natural variation of dark circles over time. Figure 6 shows the variation of clinical scoring in $\mathrm{Z} 2$ for the nine subjects over the 
Without Dark Circles - Z2

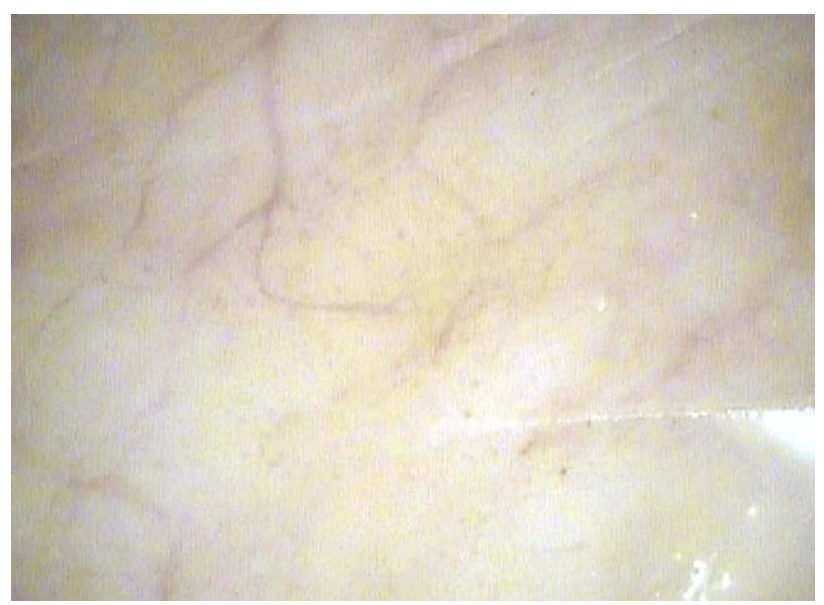

With Dark Circles - Z2

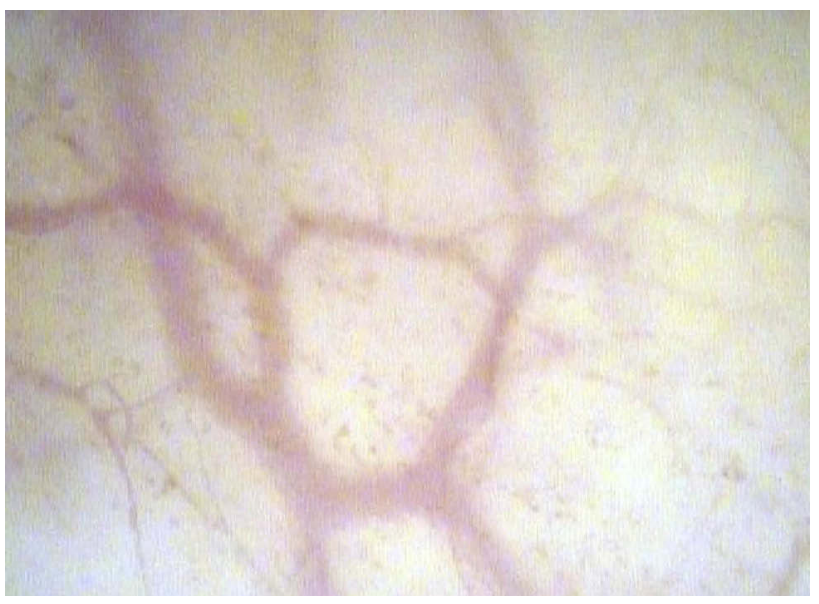

Figure 5 Example videocapillaroscopy images from subjects with and without dark circles showing visual differences in the vascular network.

Abbreviation: Z2, Zone 2.

seven days and six assessment time-points. The data clearly show the variability of dark circles over time, with many of the subjects having greater than a one grade change in score over the six time-points. While the clinical score of two of the subjects was relatively stable over time (Figure 6, S6 and $\mathrm{S} 10)$, overall there was a significant difference between the maximum and minimum score across the entire population in $\mathrm{Z} 2$, as well as in $\mathrm{Z} 1$ and $\mathrm{Z} 3$. This variation was confirmed by data from colour analysis of both VISIA and multispectral images. In addition, significant differences were also observed for both intra-day and inter-day variation (data not shown).

\section{Discussion}

The aetiology of infraorbital dark circles is complex and multi-factorial, and in addition, dark circles are difficult to measure and quantify. While they are not a cause of medical concern, it is important that efforts are made to accurately assess dark circles so appropriate treatments can be prescribed to alleviate this aesthetically unpleasing condition. The intricate anatomy and physiology of the orbital area further add to the complexity, and other studies have outlined the importance of assessing multiple zones of the infraorbital region. ${ }^{16}$ Therefore, in this research, we assessed three zones initially, before focussing on zone 2 in the middle of the lower eye contour, to gain a comprehensive understanding of the aetiology of dark circles.

Throughout this research, subjects were selected according to the absence or presence of dark circles. However, it became clear that even subjects without evident dark circles had some darkening towards the inner corner of the eye, and it was difficult to recruit subjects completely lacking in some evidence of dark circles. In fact, rather than two discrete groups, there was more or less a continuum of values, which we believe is a more accurate representation of the population. Despite these observations, the results presented here clearly distinguish between groups without evident dark circles and with dark circles. In addition, age did not appear to be a factor; there

Table 3 Summary Of Instrumental Measurements ( \pm Standard Deviation) In Caucasian Subjects With And Without Dark Circles

\begin{tabular}{|l|l|l|l|l|l|l|l|l|l|}
\hline \multirow{2}{*}{ Instrument } & Parameter & \multicolumn{2}{|l|}{ Without Dark Circles } & \multicolumn{3}{l|}{ With Dark Circles } \\
\cline { 3 - 9 } & & $\mathbf{Z C}$ & $\mathbf{Z 3}$ & $\mathbf{Z 2}$ & $\mathbf{Z I}$ & $\mathbf{Z I}$ & $\mathbf{Z 2}$ & $\mathbf{Z 3}$ & $\mathbf{Z C}$ \\
\hline \multirow{2}{*}{ VISIA } & L* & $67.1 \pm 3.0$ & $63.4 \pm 2.8$ & $58.4 \pm 4.3$ & $43.4 \pm 6.2$ & $36.1 \pm 6.5$ & $50.1 \pm 6.7$ & $57.5 \pm 6.0$ & $64.1 \pm 4.9$ \\
& $\mathrm{a}^{*}$ & $15.7 \pm 2.3$ & $12.3 \pm 2.3$ & $12.4 \pm 2.4$ & $16.7 \pm 2.4$ & $18.8 \pm 2.2$ & $16.7 \pm 3.6$ & $15.0 \pm 2.6$ & $16.9 \pm 3.4$ \\
& $\mathrm{~b}^{*}$ & $20.8 \pm 3.3$ & $19.1 \pm 3.3$ & $18.3 \pm 4.3$ & $19.0 \pm 3.6$ & $19.8 \pm 2.2$ & $21.3 \pm 4.2$ & $22.8 \pm 2.8$ & $24.2 \pm 3.0$ \\
\hline \multirow{2}{*}{ Multispectral Imaging } & Dark Circles nCDA & $0.19 \pm 0.08$ & $0.50 \pm 0.07$ & $0.62 \pm 0.08$ & $0.72 \pm 0.15$ & $0.83 \pm 0.14$ & $0.77 \pm 0.12$ & $0.65 \pm 0.09$ & $0.22 \pm 0.12$ \\
& Melanin Index & $0.17 \pm 0.19$ & $0.24 \pm 0.18$ & $0.30 \pm 0.22$ & $0.41 \pm 0.23$ & $0.54 \pm 0.25$ & $0.61 \pm 0.32$ & $0.54 \pm 0.24$ & $0.36 \pm 0.20$ \\
& Haemoglobin Index & $0.35 \pm 0.08$ & $0.43 \pm 0.07$ & $0.50 \pm 0.06$ & $0.58 \pm 0.12$ & $0.72 \pm 0.15$ & $0.72 \pm 0.16$ & $0.54 \pm 0.11$ & $0.34 \pm 0.16$ \\
\hline \multirow{2}{*}{ Ultrasound } & Skin Thickness (mm) & $1.2 \pm 0.3$ & nd & $1.2 \pm 0.3$ & nd & nd & $0.9 \pm 0.2$ & nd & $1.1 \pm 0.2$ \\
\hline
\end{tabular}

Abbreviations: ZI, Zone I; Z2, Zone 2; Z3, Zone 3; ZC, Cheek Control Zone; L*, lightness; a*, red-green component; b*, yellow-blue component; nCDA, normalized canonical discriminant analysis; nd, not determined. 


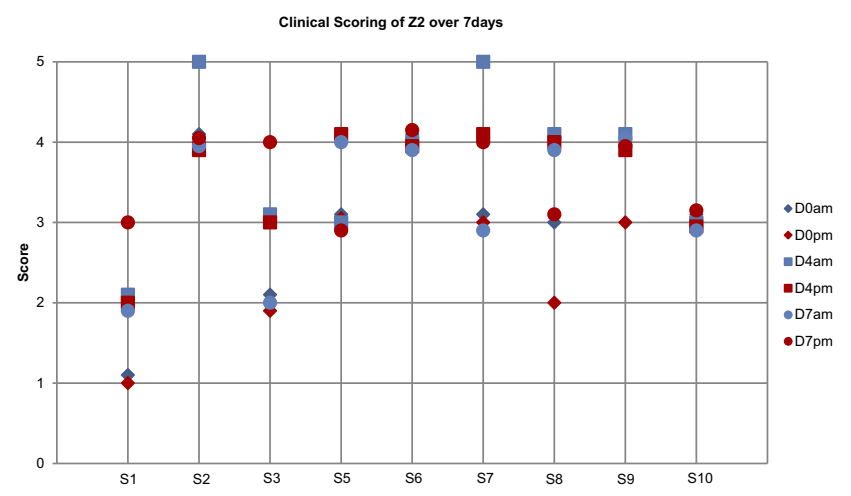

Figure 6 Clinical scoring of Zone 2 on nine subjects with dark circles over seven days.

Abbreviations: Z2, Zone 2; S, subject; D0, day 0; D4, day 4; D7, day 7; am, morning (blue); pm, afternoon (red).

was no significant difference in the age range between the two groups.

The results show that clinical scoring and $\mathrm{L}^{*}$ were well correlated. Assessments performed by spectrocolorimetry, chromametry and image processing of VISIA photographs show that dark circles are characterized by darker skin, particularly in the middle of the lower eye contour. Assessments performed with the spectrocolorimeter and the chromameter were less correlated with clinical scoring than those for VISIA image processing. This could be explained by the difficulty in accurately handling these devices in the eye area, particularly due to the size of the devices. This coupled with the complex anatomy of the orbital region may prevent close contact between the measurement head and the skin. Image processing of VISIA photographs was determined to be the most relevant for colour analysis, with the added advantage of being able to more precisely select an area of interest for analysis.

Multispectral imaging was found to be a useful technique for the assessment of dark circles and provided some valuable insights into the aetiology of dark circles. This technology allows the user to select specific wavelength to assess the presence of melanin and haemoglobin, as well as performing nCDA of the overall dark circle area. ${ }^{17}$ Some published articles have already highlighted the benefit of this technique to assess skin colour, ${ }^{18}$ and even more specifically, skin pigment, ${ }^{19}$ erythema, ${ }^{20}$ melanin, total haemoglobin and oxygen saturation in dark circles. ${ }^{21}$ The multispectral analysis performed in this research showed a higher melanin index that suggests hyperpigmentation, and a higher haemoglobin index that supports the hypothesis of blood vessel dilation in the dark circles area. Preliminary data using videocapillaroscopy to assess the vascular network also suggest there is increased dilation of blood vessel and/or an increased number of capillaries in subjects with dark circles. While further work is required to confirm these observations, this combined with a higher haemoglobin index provided strong evidence of the involvement of the vasculature in dark circles aetiology. This may be associated with inflammation in the eye area, as vascular changes and increased blood flow have been linked to the inflammatory response. ${ }^{22}$ In addition, inflammation of the skin may also trigger hyperpigmentation. ${ }^{6,23}$

Results from ultrasound imaging suggest that under-eye dark circles are linked to thinner skin in the under-eye area, which is in agreement with the work of Ohshima and Takiwaki. ${ }^{17}$ Thinner skin under the eye undoubtedly contributes to the visual appearance of dark circles, as increased blood vessel dilation and hyperpigmentation would be more evident through thinner skin.

The data on ethnic skin tones showed similar trends to those observed for Caucasian skin, though statistically significant differences between groups were not achieved for all parameters measured due to the lower number of subjects involved. Further work is certainly required to fully characterize dark circles in ethnic skin tones, particularly as genetics is suspected to play a role. It would also be interesting to compare dark circles across the ethnic skin tones to determine if phototype is linked to specific characteristics of the dark circles. The ability to perform such analysis in this study is limited by the sample size and further data is required, particularly from higher phototypes.

Finally, our results have shown the variability of dark circle intensity over time, with intra-individual, intra-day and inter-day variability observed. These results highlight the importance of performing intra-individual assessments at the same time of the day if multiple time-points are involved over an extended period of time, for example, if assessing treatment efficacy.

\section{Conclusion}

The results of this research clearly show that under-eye dark circles are characterized by darker skin, with higher colour deviations between the dark circle and surrounding areas of skin, as reflected by clinical scoring and image analysis. This work shows that clinical scoring and colour analysis of images captured with the VISIA are useful techniques to assess and track dark circles, with good correlation between these two techniques. Because of the complexity of dark circles, further work is still required to fully elucidate all the factors that contribute to their occurrence. In addition, it may be worth exploring additional techniques for 
characterization of dark circles, for example, construction of a validated photographic grading scale and more in-depth multispectral image analysis. The research presented here has, however, further built our scientific understanding and has identified three key physiological factors associated with the occurrence of infraorbital dark circles; hyperpigmentation, a tendency for more dilated, thicker or increased number of capillaries and thinner skin in the under-eye area. This combination of factors is a robust indicator of the presence of dark circles and these insights will inform future strategies for the cosmetic treatment of this issue across multiple different skin tones.

\section{Acknowledgments}

The authors thank Professor Philippe Humbert, Adeline Jeudy and Thomas Lihoreau for their contribution to this work.

\section{Disclosure}

This was biomedical research that did not involve any product as mentioned in Article L. 5311-1 of the French Public Health Code (physiology). No experimental products were evaluated. Consequently, these studies did not fall within the scope of French law, relating to public health policy $N^{\circ}$ 2004-806 of 9 August 2004 LOSP, on persons engaged in biomedical research. Moreover, these studies were conducted between 2010 and 2012 in healthy volunteers, without invasive techniques. They were thus not considered as interventional studies and were not concerned by the French legislation governing these types of studies (directive 2001/20/CE, decree dated 27 April 2006). The research was funded by Walgreens Boots Alliance. The authors report no other conflicts of interest in this work.

\section{References}

1. Freitag FM, Cestari TF. What causes dark circles under the eyes? J Cosmet Dermatol. 2007;6(3):211-215. doi:10.1111/j.1473-2165.2007.00324.x

2. Roh MR, Chung KY. Infraorbital dark circles: definition, causes and treatment options. Dermatol Surg. 2009;35(8):1163-1171. doi:10.111 1/j.1524-4725.2009.01213.x

3. Moraes Souza D, Ludtke C, Rios De Moraes Souza E, Melchiades Pinheiro Scandura K, Blessmann Webe M. Periorbital hyperchromia. Surg Cosmet Dermatol. 2011;3(3):233-239.

4. Friedmann DP, Goldman MP. Dark circles: etiology and management options. Clin Plast Surg. 2015;42(1):33-50. doi:10.1016/j.cps.2014.08. 007
5. Matsui MS, Schalka S, Vanderover G, et al. Physiological and lifestyle factors contributing to risk and severity of peri-orbital dark circles in the Brazilian population. An Bras Dermatol. 2015;90 (4):494-503. doi:10.1590/abd1806-4841.20153520

6. Sarkar R, Ranjan R, Garg S, Garg V, Sonthalia S, Bansal S. Periorbital hyperpigmentation: a comprehensive review. $J$ Clin Aesthet Dermatol. 2016;9(1):49-55.

7. Sarkar R, Das A. Periorbital hyperpigmentation: what lies beneath? Ind Dermatol Online J. 2018;9(4):229-230. doi:10.4103/idoj.IDOJ_ 303_17

8. Gendler EC. Treatment of periorbital hyperpigmentation. Aesthetic Surg J. 2005;25(6):618-624. doi:10.1016/j.asj.2005.09.018

9. Taskin B. Periocular pigmentation: overcoming the difficulties. J Pigment Disorders. 2015;2:159.

10. Daroach M, Kumaran MS. Periorbital hyperpigmentation - an overview of the enigmatous condition. Pigment Int. 2018;5:1-3. doi:10.4103/Pigmentinternational.Pigmentinternational_7_18

11. Park KY, Kwon HJ, Youn CS, Seo SJ, Kim MN. Treatments of infra-orbital dark circles by various etiologies. Ann Dermatol. 2018;30(5):522-528. doi:10.5021/ad.2018.30.5.522

12. Vrcek I, Ozgur O, Nakra T. Infraorbital dark circles: a review of the pathogenesis, evaluation and treatment. J Cutan Aesthet Surg. 2016;9 (2):65-72. doi:10.4103/0974-2077.184046

13. Chopra K, Calva D, Sosin M, et al. A comprehensive examination of topographic thickness of skin in the human face. Aesthetic Surg $J$. 2015;35(8):1007-1013. doi:10.1093/asj/sjv079

14. Huang YL, Chang SL, Ma L, Lee MC, Hu S. Clinical analysis and classification of dark eye circle. Int J Dermatol. 2014;53(2):164-170. doi:10.1111/j.1365-4632.2012.05701.x

15. Park SR, Kim HJ, Park HK, et al. Classification by causes of dark circles and appropriate evaluation method of dark circles. Skin Res Technol. 2016;22(3):276-283. doi:10.1111/srt.12258

16. Niforos F, Liew S, Acquilla R. et al. Creation and validation of a photonumeric scale to assess volume deficiency in the infraorbital region. Dermatol Surg. 2017;43(5):684-691. doi:10.1097/DSS.00000 00000001088

17. Ohshima H, Takiwaki H. Evaluation of dark circles of the lower eyelid: comparison between reflectance meters and image processing and involvement of dermal thickness in appearance. Skin Res Technol. 2008;14(2):135-141. doi:10.1111/j.1600-0846.2007.00277.x

18. Stamatas GN, Balas CJ, Kollias N. Hyperspectral image acquisition and analysis of skin. Proc SPIE Spectral Imaging Inst Appl Anal II. 2003;4959. doi:10.1117/12.479491

19. Seroul P, Hebert M, Cherel M, Vernet R, Clerc R, Jomier M. Modelbased skin pigment cartography by high-resolution hyperspectral imaging. J Electronic Imaging. 2016;69(6):48-56.

20. Madooei A, Abdlaty R, Doerwald-Munoz L, et al. Hyperspectral image processing for detection and grading of skin erythema. Proc SPIE Med Imaging. 2017:1013322. doi:10.1117/12.2254132

21. Nkengne A, Robic J, Seroul P, Gueheunneux S, Jomier M, Vie K. SpectraCam: a new polarized hyperspectral imaging system for repeatable and reproducible in vivo skin quantification of melanin, total hemoglobin, and oxygen saturation. Skin Res Technol. 2018;24 (1):99-107. doi:10.1111/srt.12396

22. Pober J, Sessa W. Inflammation and the blood microvascular system. Cold Spring Harb Perspect Biol. 2015;7:a016345. doi:10.1101/ cshperspect.a016345

23. Davis E, Callender V. Postinflammatory hyperpigmentation; a review of the epidemiology, clinical features and treatment options in skin of color. J Clin Aesthet Dermatol. 2010;3(7):20-31. 


\section{Publish your work in this journal}

Clinical, Cosmetic and Investigational Dermatology is an international, peer-reviewed, open access, online journal that focuses on the latest clinical and experimental research in all aspects of skin disease and cosmetic interventions. This journal is indexed on CAS.
The manuscript management system is completely online and includes a very quick and fair peer-review system, which is all easy to use. Visit http://www.dovepress.com/testimonials.php to read real quotes from published authors.

Submit your manuscript here: https://www.dovepress.com/clinical-cosmetic-and-investigational-dermatology-journal 